\title{
Classical phenylketonuria in Bulgaria: RFLP haplotypes and frequency of the major mutations
}

\author{
L Kalaydjieva, B Dworniczak, C Aulehla-Scholz, I Kremensky, J Bronzova, A Eigel, J Horst
}

\begin{abstract}
RFLP haplotypes and common mutations in the phenylalanine hydroxylase gene have been studied in a group of 29 Bulgarian PKU families. Haplotype distribution differs from that in other European populations, with a predominance of haplotypes 2 and 6 and a total absence of haplotype 3. The amino acid substitution in codon $\mathbf{4 0 8}$ is the most frequent molecular defect. The splicing defect in intron 12 is not found in Bulgarian PKU patients. Testing for three mutations, reported to be common among haplotype 1 and 4 alleles, has shown that they occur less frequently in Bulgarian PKU patients. Screening with five pairs of allele specific oligonucleotides failed to show the mutation in $59 \%$ of the patients. These findings add to the evidence that PKU is heterogeneous and that significant interpopulation differences exist. At present, DNA data cannot be used as an aid in early clinical classification and prognosis of hyperphenylalaninaemia in Bulgaria.
\end{abstract}

Neonatal screening in Bulgaria, with nearly one million neonates tested, has shown an incidence of classical phenylketonuria (PKU) of 1 in 21000 and a relatively high frequency of mild hyperphenylalaninaemia (HPA) with a PKU/HPA ratio of $1 / 2$. Experience has shown that both parental anxiety and non-compliance with the diet are frequent problems which require prompt classification of the phenylalaninaemias detected by the screening.

Studies of DNA polymorphisms in Danish HPA families established a correlation between the patients'

Laboratory of Molecular Pathology, Medical Academy, Sofia, Bulgaria.

L Kalaydjieva, I Kremensky, J Bronzova

Institut für Humangenetik, Vesaliusweg 12-14, Münster 4400, FRG.

B Dworniczak, C Aulehla-Scholz, A Eigel, J Horst

Correspondence to Professor Horst.

Received for publication 21 June 1990.

Accepted for publication 25 June 1990 phenotypes and their restriction fragment length polymorphism (RFLP) haplotypes. ${ }^{1}$ This finding, and complementary studies of $\beta$ thalassaemia, ${ }^{2}$ suggested linkage between RFLP haplotypes and specific mutations in the phenylalanine hydroxylase (PAH) gene. The finding of a $G$ to $A$ transition at the splice donor site of intron 12 in haplotype 3 chromosomes, ${ }^{3}$ and of an amino acid substitution in codon 408 of the PAH gene linked to haplotype $2,{ }^{4}$ gave credence to the above suggestions. These findings also indicated that PKU is genetically homogeneous and that the 'founder effect' played a major role in its spread. However, more recent data from other European populations showed a variable pattern of haplotypes and mutations and suggested that PKU is more heterogeneous than was initially thought. ${ }^{5-7}$

This work aimed to study the molecular basis of phenylketonuria in Bulgaria in comparison with other Caucasian populations and to evaluate the feasibility of using DNA data in early clinical classification and prognosis.

\section{Materials and methods}

PATIENTS

The study included 29 PKU patients and 50 heterozygous parents, that is, 58 mutant and 50 normal alleles. Eleven patients were of Turkish ethnic background. The patients were classified as having classical PKU using the criteria described by Güttler, ${ }^{8}$ namely neonatal phenylalanine levels $>1200 \mu \mathrm{mol} / \mathrm{l}$, residual PAH activity below $1 \%$ (calculated from the protein loading data as described by Trefz et $a l^{9}$ ), and phenylalanine tolerance less than $20 \mathrm{mg} / \mathrm{kg}$ (data on tolerance are not yet complete in patients under 5 years of age). In cases detected late, high blood phenylalanine and the presence of severe mental retardation served as the main criteria.

\section{METHODS}

DNA was isolated from peripheral leucocytes as described previously. ${ }^{10}$ For haplotype analysis, DNA samples were digested with BglII, PvuII, $E c o$ II, MspI, XmnI, HindIII, and EcoRV. Southern 
blot analysis was performed as described previously ${ }^{11}$ using ${ }^{32} \mathrm{P}$ labelled cDNA probe phPAH $247 .{ }^{12}$

The samples were screened for the presence of the following mutations previously reported to occur in West European populations: exon 5, codon 158 $(\mathrm{CGG} \rightarrow \mathrm{CAG})^{13}{ }^{14}$; exon 7 , codon $261(\mathrm{CGA} \rightarrow \mathrm{CAA})^{14}$; exon 7 , codon $281(C C T \rightarrow C T T)^{15}$; exon 12 , codon 408 (CGG $\rightarrow$ TGG) ${ }^{4}$; exon $12,5^{\prime}$ splice donor site $(\mathrm{GT} \rightarrow \mathrm{AT}) .^{3}$ The presence of each mutation was tested after amplification of exons 5, 7, and 12 and the flanking intronic sequences in the PAH gene. PCR conditions were as described previously. ${ }^{16}$ Dot blot hybridisation with ${ }^{32} \mathbf{P}$ labelled allele specific oligonucleotides (ASO) was used to detect the presence of the mutations. ${ }^{17}$ The sequences of the oligonucleotide probes were as described. ${ }^{3414} 15$ Previously studied patients of German origin, 51215 whose genotypes had been confirmed by direct sequencing, served as positive and negative controls.

\section{Results}

\section{RFLP HAPLOTYPES}

Haplotype assignment was possible in 25 out of 29 families. The RFLP data are shown in table 1. Seven different RFLP haplotypes were found in the group of mutant alleles. Haplotype 2 was the most common and occurred in $38 \%$ of mutant alleles. Second in frequency was haplotype $6(22 \%)$, which was found mainly in patients of Turkish origin. These two

Table 1 Percentage distribution of RFLP haplotypes in PKU and in normal alleles in Bulgarian families.

\begin{tabular}{ccc}
\hline Haplotype & $\begin{array}{c}\text { PKU alleles } \\
(\mathbf{n}=50)\end{array}$ & $\begin{array}{c}\text { Normal alleles } \\
(\mathbf{n}=42)\end{array}$ \\
\hline 1 & 16 & 24 \\
2 & 38 & 2 \\
3 & -10 & 2 \\
4 & 2 & 15 \\
5 & 22 & 2 \\
6 & -8 & 2 \\
7 & 4 & 10 \\
10 & - & 5 \\
32 & & 38 \\
\hline
\end{tabular}

Haplotype classification is as described. ${ }^{11}$ haplotypes were in linkage disequilibrium with the PKU gene $\left(\chi^{2}=14.99, \mathrm{p}<0.001\right.$ for haplotype 2 and $\chi^{2}=6.11, p<0.05$ for haplotype 6). Haplotypes 1 and 4 were also found to be relatively common and occurred in mutant chromosomes with frequencies similar to those seen in normal alleles. Haplotype 3 was absent altogether in the group studied. Eight out of 25 patients were haplotype homozygotes.

The polymorphic characteristics of normal chromosomes were heterogeneous, with a prevalence of haplotypes $1(24 \%)$ and $4(15 \%)$. In the 42 normal alleles where haplotype assignment was possible, a total of 20 different haplotypes was found, most of which occurred in single chromosomes.

\section{MUTATION ANALYSIS}

All 29 patients (58 PKU chromosomes) were screened for five molecular defects reported to be common or relatively common in other European populations. The data are shown in table 2.

The $C$ to $T$ transition in codon 408 , resulting in a Trp for Arg substitution, was found to be the major molecular defect which occurred in $34 \%$ of all PKU alleles studied. This mutation was found in all haplotype 2 alleles and also in a single haplotype 5 chromosome.

The splicing defect in intron 12, which accounts for nearly $\mathbf{4 0 \%}$ of HPA alleles in Denmark, ${ }^{3}$ was absent in Bulgarian PKU patients. This is in agreement with the absence of haplotype 3, which has been reported to be in tight linkage with the splicing mutation. ${ }^{3}$

The missense mutation in exon 5, codon 158 (Arg $\rightarrow$ Gln), which constitutes about $40 \%$ of mutant haplotype 4 alleles in western Europeans, ${ }^{13}{ }^{14}$ was detected in a single chromosome in the present study. Haplotype assignment was not fully possible in this family, but informative RFLPs (BglII, PvuIIa, and PvuIIb) were compatible with haplotype 4 , and the polymorphic AluI site in exon 7 which is typical of haplotype $4^{18}$ was also present.

Another missense mutation, the $G$ to $A$ transition in codon 261 (Arg $\rightarrow \mathrm{Gln}$ ), was carried by two mutant chromosumes, both haplotype 1 . Thus the mutation in codon 261 is similar in frequency to other European populations. ${ }^{14}$ In vitro expression studies have shown

Table 2 Mutations found in Bulgarian PKU patients.

\begin{tabular}{lcccrrr}
\hline Haplotype & Cod158 & Cod261 & Cod281 & Cod408 & Unknown & Total \\
\hline 1 & & 2 & 1 & 19 & 5 & 8 \\
2 & 1 & & 1 & 5 & 19 \\
4 & & & & 11 & 11 \\
5 & & & 4 & 2 \\
10 & & & & 7 & 7 \\
32 & & & & & 7 \\
Not assignable & & & & & & \\
\hline
\end{tabular}


that this defect results in no detectable loss of activity. ${ }^{14}$ In the present study both patients with $\mathrm{Arg}^{261} \rightarrow \mathrm{Gln}^{261}$ substitution were compound heterozygotes, for the common defect in codon 408 in one case and for an unknown defect linked to haplotype 6 in the other. Although phenylalanine tolerance has not been established yet, both patients had high neonatal phenylalanine levels, a protein loading curve with sustained high phenylalanine levels, and residual PAH activity below $1 \%$.

The amino acid substitution in codon 281 (Pro $\rightarrow$ Leu) was detected in a single haplotype 1 chromosome. This defect has been reported to account for over $25 \%$ of mutant haplotype 1 alleles in the German population. ${ }^{15}$

\section{Discussion}

Both RFLP haplotypes and mutations in Bulgarian PKU patients differ in distribution from other Caucasian populations.

About $90 \%$ of the PKU alleles in northern European populations are confined to haplotypes 1 to $4,{ }^{15-7}$ whereas in Bulgarian PKU patients these haplotypes account for $64 \%$ of mutant alleles. The difference is mainly related to the total absence of haplotype 3, which is very common in Denmark ${ }^{1}$ and gradually declines in frequency in the south and east of Europe.

A significant proportion of PKU alleles in this study belong to haplotype 6 . This polymorphic haplotype is almost absent in northern Europeans, but has been reported to occur frequently in Turkish PKU patients. ${ }^{19}$ Haplotype 6 alleles accounted for about $30 \%$ of chromosomes with an unknown mutation in this study. This haplotype is in linkage disequilibrium with the disease and may therefore be associated with a single molecular defect.

As in other Caucasian populations, ${ }^{15-7}$ haplotypes 1 and 4 were found to be relatively common in Bulgarian PKU patients. Screening for three mutations, which have been previously detected in Caucasian chromosomes of these haplotypes, failed to show the molecular defect responsible in nearly $70 \%$ of these alleles. The lack of linkage disequilibrium between these haplotypes and PKU is a consistent finding in all European populations. This fact and the existence of interpopulation differences shown in this study suggest that haplotypes 1 and 4 may be very heterogeneous, not only in terms of clinical phenotype, but also in terms of molecular defects leading to classical PKU.

The present findings are further evidence of the heterogeneous molecular basis of phenylketonuria and suggest that various mechanisms might have played a role in its spread. Unlike the common mutations in codon 408 and in the $5^{\prime}$ splicing site of intron 12 , which probably spread through founder effect, mutations linked to haplotypes 1 and 4 can be expected to be heterogeneous and to vary between populations.

This study also indicates that the use of molecular genetics as an aid to early clinical diagnosis is not feasible in Bulgarian PKU families at present. The use of five pairs of ASOs has shown both mutations in five out of 29 patients, four homozygous for the defect in codon 408 and one compound heterozygote (cod261/cod408). If haplotype 6 is indeed associated with a single mutation, as suggested by the presence of linkage disequilibrium, the proportion of informative cases will increase when this mutation becomes known; two patients in the present study are haplotype 6 homozygotes and three are 2/6 compounds. In the meantime, haplotype 6 in the homozygous state or in combination with haplotype 2 can be tentatively regarded as indicative of classical PKU, especially in patients of Turkish origin.

Although PKU is a treatable condition, our experience has shown that the majority of families are interested in prenatal diagnosis in future pregnancies. Taking into account the limited number of cases where direct mutation detection is applicable ( $17 \%$ of families are fully informative and $45 \%$ are partially informative), RFLP analysis has to be regarded as the main diagnostic tool. Most PKU carriers are RFLP heterozygotes, which results in almost $90 \%$ of families being fully informative for fetal RFLP analysis.

This study was supported by the Deutsche Forschungsgemeinschaft, the Bundesministerium für Forschung und Technologie, and by the Bulgarian Neurosciences and Behaviour Research Programme. LK was a fellow of the Alexander von Humboldt Foundation. The cDNA probe hpPAH247 was kindly provided by Dr S L C Woo.

1 Chakraborty R, Lidksy AS, Daiger SP, et al. Polymorphic DNA haplotypes at the human phenylalanine hydroxylase locus and their relationship with phenylketonuria. Hum Genet 1987;76: 40-6.

2 Orkin SH, Kazazian HH, Antonarakis SE, et al. Linkage of $\beta$ thalassemia mutations with DNA polymorphisms in the human $\beta$-globin cluster. Nature 1982;296:627-31.

3 DiLella AG, Marvit J, Lidsky AS, et al. Tight linkage between a splicing mutation and a specific DNA haplotype in phenylketonuria. Nature 1986;322:799-803.

4 Dilella AG, Marvit J, Brayton K, Woo SLC. An amino acid substitution involved in phenylketonuria is in linkage disequilibrium with DNA haplotype 2 . Nature 1987;327:333-6.

5 Aulehla-Scholz C, Vorgert M, Sautter E, et al. Phenylketonuria: a distribution of DNA diagnostic patterns in German families. Hum Genet 1988;78:353-5.

6 Rey F, Berthelon M, Caillaud C, et al. Clinical and molecular heterogeneity of phenylalanine hydroxylase deficiencies in France. Am 7 Hum Genet 1988;43:914-21.

7 Sullivan SE, Moore SD, Connor JM, et al. Haplotype distribution of the human phenylalanine hydroxylase locus in Scotland and Switzerland. Am F Hum Genet 1989;44:652-9.

8 Güttler F. Hyperphenylalaninemia: diagnosis and classification of the various types of phenylalanine hydroxylase deficiency in childhood. Acta Paediatr Scand 1980;280(suppl): 1-80.

9 Trefz FK, Schmidt H, Bartholomé K, et al. Differential diagnosis and significance of various hyperphenylalaninemias. In: Bickel 
H, Wachtel U, eds. Inherited diseases of amino acid metabolism. Stuttgart: Thieme, 1985: 86-100.

10 Old J. Fetal DNA analysis. In: Davies K, ed. Human genetic diseases a practical approach. Oxford: IRL Press, 1986:1-19.

11 Lidsky AS, Ledley FD, DiLella AG, et al. Extensive restriction site polymorphism at the human phenylalanine hydroxylase locus and application in prenatal diagnosis of phenylketonuria. Am $\mathcal{F}$ Hum Genet 1985;37:619-34.

12 Kwok S, Ledley FD, Robson JH, Woo SLC. Nucleotide sequence of a full-length cDNA clone of human phenylalanine hydroxylase. Biochemistry 1985;24:556-61.

13 Dworniczak B, Aulehla-Scholz C, Horst J. Phenylketonuria: detection of a frequent haplotype 4 allele mutant. Hum Genet $1989 ; 84: 95-6$.

14 Okano Y, Wang T, Eisensmith RC, et al. Missense mutations associated with RFLP haplotypes 1 and 4 of the human phenylalanine hydroxylase gene. Am $\mathcal{f}$ Hum Genet 1990;46: 18-25.
15 Dworniczak B, Grudda K, Stümper J, Bartholomé K, AulehlaScholz C, Horst J. Phenylalanine hydroxylase gene: identification of a new frequent missense mutation causing severe phenylketonuria. Genomics (submitted).

16 Spiegelberg R, Aulehla-Scholz C, Erlich H, Horst J. A Bthalassemia gene caused by a 290-base pair deletion: analysis by direct sequencing of enzymatically amplified DNA. Blood 1989;73:1695-8.

17 DiLella AG, Huang WM, Woo SLC. Screening for phenylketonuria mutations by DNA amplification with the polymerase chain reaction. Lancet 1988;i:497-9.

18 Dworniczak B, Aulehla-Scholz C, Horst J. Phenylalanine hydroxylase gene: silent mutation uncovers evolutionary origin of different alleles. Clin Genet (in press).

19 Lichter-Konecki U, Schlotter M, Yalak C, et al. DNA haplotype analysis at the phenylalanine hydroxylase locus in the Turkish population. Hum Genet 1989;81:373-6. 